\title{
Presence of Obstetrical Conditions among Women with Pelvic Inflammatory Diseases: Experience of 50 Cases in Bangladesh
}

\author{
Tahamina Khanum ${ }^{1 *}$, Gulshan Ara1 ${ }^{1}$ Zubayer Miah², Abdullah Yusuf ${ }^{3}$ \\ ${ }^{1}$ Department of Gynaecology \& Obstetrics, Enam Medical College, Dhaka, Bangladesh \\ ${ }^{2}$ Department of Psychiatry, Shaheed Tajuddin Ahmad Medical College, Gazipur, Bangladesh \\ ${ }^{3}$ Department of Microbiology, National Institute of Neurosciences \& Hospital, Dhaka, Bangladesh \\ Email: ^drtahamina75@gmail.com,dr.gulshanara1995@gmail.com, zubayer_miah@yahoo.com, ayusuf75@yahoo.com
}

\author{
How to cite this paper: Khanum, T., Ara, \\ G., Miah, Z. and Yusuf, A. (2018) Presence \\ of Obstetrical Conditions among Women \\ with Pelvic Inflammatory Diseases: Expe- \\ rience of 50 Cases in Bangladesh. Open \\ Journal of Obstetrics and Gynecology, 8, \\ 391-399. \\ https://doi.org/10.4236/ojog.2018.84044 \\ Received: March 7, 2018 \\ Accepted: April 20, 2018 \\ Published: April 23, 2018 \\ Copyright $\odot 2018$ by authors and \\ Scientific Research Publishing Inc. \\ This work is licensed under the Creative \\ Commons Attribution-NonCommercial \\ International License (CC BY-NC 4.0). \\ http://creativecommons.org/licenses/by-nc/4.0/ \\ (c) (i) \&) Open Access
}

\begin{abstract}
Background: Pelvic Inflammatory Diseases can be associated with the different obstetrical condition. Objectives: The purpose of the present study was to see the Presence of Obstetrical Conditions among Women with pelvic inflammatory diseases (PID). Methodology: This cross-sectional of study was conducted in the Department of Obstetrics and Gynaecology at Dhaka Medical College, Dhaka from January 2007 to June 2007 for a period of six (6) months. Women with the age group of $15-45$ years presented with lower abdominal pain, tenderness, per vaginal discharge and cervical motion tenderness were included in this study. A pre-designed data collection sheet was used to record the history of different obstetrical condition. Result: A total number of 50 women presented with PID were recruited for this study of which majority was in para 2 to 5 which was $30(60.0 \%)$ cases. Majority of the patients had given the history of home delivery $(74.0 \%)$ which was performed by untrained birth attendance $(60.0 \%)$. The history of induced abortion was given by $24.0 \%$ cases. History of more than 2 times menstrual regulation (MR) and 2 to 5 times were performed in $20(40.0 \%)$ cases in each. Among 50 cases majority used intrauterine device (IUCD) which were 20 (40.0\%) cases. Conclusion: In conclusion majority of the women presented with PID have given the history of para 2 to 5 , home delivery with untrained birth attendance, induction abortion and history of more than two times MR.
\end{abstract}

\section{Keywords}

Obstetrical Conditions, Home Delivery, Pelvic Inflammatory

Diseases, MR 


\section{Introduction}

Pelvic inflammatory disease (PID) is an infection-caused inflammatory continuum from the cervix to the peritoneal cavity [1]. Most importantly, it is associated with fallopian tube inflammation, which can lead to infertility, ectopic pregnancy, and chronic pelvic pain [2]. According to a national estimate, in 2001 more than 750,000 cases of pelvic inflammatory disease occurred in the United States; however, over the past two decades, the rates and severity of pelvic inflammatory disease have declined in North America and Western Europe [3] [4] [5]. Despite progress, however, pelvic inflammatory disease remains a problem because reproductive outcomes among treated patients are still suboptimal, subclinical pelvic inflammatory disease remains poorly controlled, and programs aimed at the prevention of pelvic inflammatory disease are not feasible in much of the developing world [6].

The microbial etiology is linked to sexually transmitted microorganisms, including Chlamydia trachomatis, Neisseria gonorrheae, Mycoplasma genitalium, and bacterial vaginosis-associated microorganisms, predominantly anaerobes [4]. Pelvic pain and fever are commonly absent in women with confirmed PID. Clinicians should consider milder symptoms such as abnormal vaginal discharge, metrorrhagia, postcoital bleeding, and urinary frequency as potential symptoms associated with the disease, particularly in women at risk of sexually transmitted infection [6]. The diagnosis of PID is based on the findings of lower genital tract inflammation associated with pelvic organ tenderness. The outpatient treatment of mild-to-moderate PID should include tolerated antibiotic regimens with activity against the commonly isolated microorganisms associated with PID and usually consists of an extended spectrum cephalosporin in conjunction with either doxycycline or azithromycin [7]. Clinically severe PID should prompt hospitalization and imaging to rule out a tuboovarian abscess. Parenteral broad-spectrum antibiotic therapy with activity against a polymicrobial flora, particularly gram-negative aerobes and anaerobes, should be implemented. Screening for and treatment of Chlamydia infection can prevent PID [8].

Pelvic inflammatory disease is now a major concern as because it can cause reproductive disability including infertility, ectopic pregnancy and chronic pelvic pain [3]. After the introduction of laparoscopy in the 1960s, research on pelvic inflammatory disease proliferated which leads to major breakthroughs in the understanding of the microbial causes of the disease and its relationship to reproductive disability, as well as enabling the standardization of antimicrobial treatment [9]. Despite progress, however, pelvic inflammatory disease remains a problem because reproductive outcomes among treated patients are still suboptimal, subclinical pelvic inflammatory disease remains poorly controlled, and programs aimed at the prevention of pelvic inflammatory disease are not feasible in much of the developing world. The reason may be due the different history of obstetrical condition [8]. In this regards this present study was undertaken to the 
presence of obstetrical conditions among women with pelvic inflammatory diseases (PID).

\section{Methodology}

This was a prospective observational type of cross-sectional of study. This study was conducted in the outpatient department (OPD) of the Department of Obstetrics and Gynaecology at Dhaka Medical College, Dhaka. The duration of study was from January 2007 to June 2007 for a period of six (6) months. Women with the age group of 15 to 45 years presented with lower abdominal pain, tenderness, per vaginal discharge and cervical motion tenderness were included in this study. Women with fibroid uterus, uterovaginal prolapsed or cystocele was excluded from this study. Clinically PID was diagnosed with the clinical features of lower abdominal pain, abdominal tenderness, per vaginal discharge and cervical motion tenderness in the women. After attending in gynaecology OPD in Dhaka Medical College \& Hospital (DMCH), Dhaka, Bangladesh an introduction was given to the patients regarding the purpose and importance of the study. After taking informed verbal consent from the patients, a pre-designed data collection sheet was used to record the history of different obstetrical condition like age of the patients, number of parity, places of delivery, types of abortion history, history of different issues related to menstrual regulation (MR) and history of uses of different types of uses of contraceptives methods. Statistical Packages for Social Sciences (SPSS) Statistics 20.0 was used for the statistical analyses. Categorical data were presented as frequency and percentage.

\section{Results}

The study was performed on 50 cases. Out of 50 cases of pelvic inflammatory disease majority (50\%) belonged to the age group of 26 to 35 years followed by more than 35 years age group and 18 to 25 Years of age group which were 14 (28.0\%) cases and $10(20.0 \%)$ cases respectively (Table 1$)$.

Among 50 women with PID majority were in para 2 to 5 which was 30 (60.0\%) cases followed by more than 5 para and para 1 which were $14(28.0 \%)$ cases and $10(20.0 \%)$ cases respectively. However, primigravida was only in 3 (6.0\%) cases (Table 2).

Among 50 cases $74 \%$ cases were underwent home delivery of which $60 \%$ labour were performed by untrained birth attendance and $14 \%$ cases were by trained birth attendance; furthermore $26 \%$ cases were given the history of hospital delivery (Table 3 ).

Out of 50 patient $24 \%$ cases were given the history of induced abortion, $14 \%$ has septic abortion, $24 \%$ spontaneous abortion and $20 \%$ had D and C (Table 4 ).

Among 50 cases more than 2 times MR and 2 to 5 times were performed in 20 $(40.0 \%)$ cases in each; however, $10(20.0 \%)$ cases were given no history of MR. Among all cases $11(22.0 \%)$ cases were performed by trained person and the rest $29(58.0 \%)$ cases were performed by untrained person (Table 5). 
Table 1. Age distribution of study population $(n=50)$.

\begin{tabular}{ccc}
\hline Age Group (years) & Frequency & Percentage \\
\hline Less than 18 Years & 1 & 2.0 \\
18 to 25 Years & 10 & 20.0 \\
26 to 35 Years & 25 & 50.0 \\
More than 35 Years & 14 & 28.0 \\
Total & $\mathbf{5 0}$ & $\mathbf{1 0 0 . 0}$ \\
\hline
\end{tabular}

Table 2. Distribution of number of parity among the study population $(n=50)$.

\begin{tabular}{ccc}
\hline Parity & Frequency & Percentage \\
\hline Primigravida & 3 & 6.0 \\
1 & 5 & 10.0 \\
2 to 5 & 30 & 60.0 \\
More Than 5 & 12 & 24.0 \\
Total & $\mathbf{5 0}$ & $\mathbf{1 0 0 . 0}$ \\
\hline
\end{tabular}

Table 3. Distribution of the places of delivery among the study population $(n=50)$.

\begin{tabular}{ccc}
\hline Place of delivery & Frequency & Percentage \\
\hline Hospital delivery & 13 & 26.0 \\
Home delivery attended by & & \\
Untrained BA & 30 & 60.0 \\
Trained BA & 7 & 14.0 \\
Puerperal sepsis & 5 & 10.0 \\
\hline
\end{tabular}

$\mathrm{BA}=$ Birth Attendance.

Table 4. Types of abortion history among the study population $(\mathrm{n}=50)$.

\begin{tabular}{cccc}
\hline Events & Less than 2 & More than 2 & Total \\
\hline Spontaneous Abortion & 5 & 7 & $12(14.0 \%)$ \\
Induced Abortion & 6 & 8 & $14(28.0 \%)$ \\
D \& C & 10 & - & $10(20.0 \%)$ \\
Septic Abortion & 4 & 10 & $14(28.0 \%)$ \\
\hline
\end{tabular}

D \& C = Dilatation and Curettage.

Table 5. Distribution of history of MR among the study population $(n=50)$.

$\begin{array}{ccc}\text { Variables } & \text { Frequency } & \text { Percentage } \\ & \text { Number of MR } & \\ 0 & 10 & 20.0 \\ \text { Less than } 2 & 20 & 40.0 \\ 2 \text { to } 5 & 20 & 40.0 \\ & \text { MR Performed by } & 22.0 \\ \text { Trained Person } & 11 & 58.0 \\ \text { Untrained Person } & 29 & \end{array}$


Among 50 cases majority were used intrauterine device (IUCD) which were $20(40.0 \%)$ cases followed by oral contraceptive pill (OCP) users, barrier users and permanent sterilization (tubectomy) which were $15(30.0 \%)$ cases, 5 (10.0\%) cases and $4(8.0 \%)$ cases respectively; however, $6(12.0 \%)$ cases were contraceptive non users (Table 6).

\section{Discussion}

In this present study 50 cases of PID patients were examined. It showed that the highest percentage (50.0\%) of PID is in the age group of 26 to 35 years. Peterson also mentioned that women with PID were usually under the age of 25 years. Shah et al. [10] showed in his study that 87 percent of the patient belong the age group of 20 - 35 years. Tarafder [11] showed the highest incidence $55.21 \%$ of PID in the age group of 26 - 35 years. Another study St John et al. [12] concluded that cases of PID were usually between the age group of 30 to 44 years (67.1\%) PID occur more in the younger age group in the Western countries, where it is mostly STD related. But in the developing countries it is more commonly in the age group and mostly related to the obstetrical events [11].

Younger age is marked by the biological characteristics favourable to develop PID, like lower prevalence of protective chlamydial antibody, longer zone of cervical ectopy and greater permeability of the cervical mucus [7]. In this study majority of the women with pelvic inflammatory disease were multipara which $60.0 \%$ cases were. However, maximum patients were delivered at home $(74.0 \%)$ and $40.0 \%$ delivery cases were conducted by untrained birth attendants; furthermore $10 \%$ patients had the history of puerperal sepsis. Similar to the present study result, Trafder [11] showed that $83.85 \%$ of the patients were multipara and $90.8 \%$ of were delivered at home. Again in this present study $67.03 \%$ cases delivery were conducted by untrained birth attendant and $56.21 \%$ were complicated by puerperal sepsis. Sultana [13] showed that $81.7 \%$ cases of PID patients were the history of home delivery and $81.0 \%$ were multipara; furthermore $73.4 \%$ of the patients had the history of puerperal sepsis which is consistent with the present study result. Perterson et al. [14] showed that PID occurred mostly in multipara. In another study De-Vries et al. [15] has shown that $74.4 \%$ of the PID cases were multiparous which are mostly in acute cases. These studies, therefore, focused that PID occurred in mostly parous women in the developing countries

Table 6. History of contraceptives among the study population $(\mathrm{n}=50)$.

\begin{tabular}{ccc}
\hline Contraceptive & Frequency & Percentage \\
\hline Pill & 15 & 30.0 \\
IUCD & 20 & 40.0 \\
Barrier & 5 & 10.0 \\
Tubectomy & 4 & 8.0 \\
Nil & 6 & 12.0 \\
\hline
\end{tabular}


and the majority were young multiparous women in developed countries.

The rural women of Bangladesh mostly depend on untrained birth attendant who conduct the delivery in a very unhygienic way [9]. There is no provision of using sterile gloves. In addition to that per vaginal examination is done repeatedly even after rupture of membrane. Sometimes they are using lubricating oil. These give the opportunity for the pathogen to enter into the upper genital tract and alter the normal sterile environment. Again, the untrained birth attendant usually perform traumatic vaginal delivery which causes perineal tear, cervical tear and also performed manual removal of placenta which favor postpartum pelvic infection [9]. However, infection initially remains localized to the pelvic region and if is not treated efficiently, this leads to chronic PID. And subsequently causes the chronic PID with its sequelae. In a maternal mortality study it has been found that $16.7 \%$ women reported symptoms of PID at postpartum period [10].

This study has shown frequency of PID among the women with the different ways of termination of pregnancy in the form of MR as well as abortion which may be induced or spontaneous. It is more common after induced abortion, spontaneous abortion without completed by Dilatation and Curettage. In this series $28.0 \%$ cases had the history of induced abortion, $24.0 \%$ had spontaneous abortion, $28.0 \%$ ended with complications by sepsis. Regarding MR it becomes evident that $40 \%$ have the history of more than two times MR and $40.0 \%$ up to 2 MR. From these finding it is obvious that PID was more common in the patients having repeated MR specially the unauthorized person which is about $58 \%$ cases. Sultana [13] showed that $72.2 \%$ patients had the history of termination of pregnancy. Tarafder [7] showed $66.13 \%$ of her patients had history of termination of pregnancy; fifty percent of them underwent puerperal sepsis. Kocher [16] showed the highest incidence of pelvic infection occurred following abortion. It is estimated that 36 to 53 million induced abortion performed through the word of which 21 million are unsafe [13].

The prevalence of pelvic infection following pregnancy termination depends upon the precaution and procedure of termination. In Bangladesh MR is mostly done by the unskilled person in unauthorized place with a very unhygienic way although it should be done by the trained personal in proper hygienic way. As a result the patients subsequent develop PID. The endometrial cavity is normally sterile. The natural barrier to pelvic infection is the cervix where downward flows of the mucous and ciliary action are reinforced by production of lysozyme. With the help of cervical secreted IgA, the lysozyme hydrolyses peptidoglycan links of microorganism allow osmotic destruction [8]. This cervical protective barrier is compromised after abortion, instrumentation like D \& C, IUCD insertion, MR and hysterosalpingography or as a result of sexual promiscuity [1].

The dramatic increase in use of contraception worldwide makes it imperative to understand the effect of contraceptives on the health of women. PID may follow elective abortion in about $10.0 \%$ of patients. Incidence of Chlamydia tra- 
chomatis screened was $14.0 \%$. This study emphasizes the frequency of PID in different methods of contraceptives. From the series it has been found that $12.0 \%$ of the patients were contraceptive non users; $30.0 \%$ cases had the history of taking oral contraceptive pill; $40.0 \%$ cases were IUCD users and $10.0 \%$ cases were barrier method users. In this study $8.0 \%$ patient had history of tubectomy. Sultana [13] showed that $48.1 \%$ patients were contraceptive non-users; however, $23.5 \%$ cases were using oral pill and $74.0 \%$ cases had the history of IUCD insertion. Tarafder [7] showed that $28.12 \%$ of the patients had the history of taking oral contraceptive pill; IUCD was on third position. Sciarra [17] showed that women with IUCD has symptom of PID which was 2.5 times of non-users and 1.8 times on women using other contraceptives. These patients also showed that a relative risk of PID of $0.3 \%$ to $0.9 \%$ for oral contraceptive users compared to non-users of contraceptives and even lower relative risk compared to users of all other method. IUCD was often regarded as important iatrogenic cause of PID. It is stated that risk is 3 to 4 folds more [16]. In FIGO manual on family planning [2], it has been mentioned that IUCD is associated with an increase risk of PID which is estimated to be 1.5 to 10 fold for IUCD users compared to other methods of contraceptives. There may be lower rate of PID among users of newer copper-bearing device. Risk of PID appears more common in the first few months following insertion and reinsertion. Women using IUCD for 5 or more years are more likely to have a more severe form of disease [2].

During IUCD insertion number of vaginal and cervical organisms enters into the endometrial cavity leading to PID. A number of different organisms are responsible like gonococcus, Chlamydia trachomatis, Mycoplasma, anaerobic and aerobic bacteria [13]. Prompt recognition and treatment should reduce the tubal damage and subsequent infertility, ectopic pregnancy. Use of doxycycline at time of IUCD insertion may reduce the risk of PID.

The relationship between oral contraceptive and PID is complex. Oral contraceptive increase cervical erosion which favours chlamydia infection. On the other hand in upper genital tract oral contraceptive provides some protection against symptomatic infection. Oral contraceptive decreases blood loss which is favorable environment for bacterial growth. Thickened cervical mucus hinders the absconding growth mechanical barrier; furthermore condom also protects the upper genital tract from STD and other ascending infections [9]. Oral contraceptive has a protective effect against PID [8].

MacLean [18] found that relative risk of PID was 0.5 with oral contraceptives and diaphragm, 0.7 with sterilization, 1.8 with medicated IUCD, 3.3 with non-medicated IUCD and 1.3 with previous IUCD user, suggesting that IUCD increases the risk of PID. Another study [9] showed the levonorgestrel releasing IUCD provides excellent contraception, may reduce the rate of PID and ectopic pregnancy compared to other copper releasing IUCD. Senarayek and Kramer [19] strongly imply that oral contraceptives have a protective effect against PID.

It is thought that oral contraceptive use has been associated with lower risk of 
PID but higher risk of chamydia cervicitis. A study showed that women with un-recognized endometritis were 4.3 times more likely to develop PID than women with recognized endometritis using oral contraceptives [20] and has concluded that future studies need to fully characterize the risk.

There are some limitations of the study. Small sample size is the major limitation. Furthermore this is a single centered study.

\section{Conclusion}

In conclusion it can be said that obstetrical events play a vital role in causation of PID. Home delivery is common to most of the patients conducted by untrained birth attendants. A good number of patients are conducted by untrained birth attendants and have the history of puerperal sepsis. It is observed that there is an increased trend of induced abortion and repeated MR performed by the unskilled person. MR and induced abortion are found as a method of termination of unplanned pregnancies rather than medically indicated, which ultimately leads to pelvic infection. Safe delivery practice by skilled personal at the community level is strongly recommended to prevent PID.

\section{Conflict of Interest}

The authors of this paper have declared that there is no conflict of interest to any of the authors.

\section{Funding Agency}

This study has been performed without any funding from outside else.

\section{References}

[1] Brunham, R.C., Gottlieb, S.L. and Paavonen, J. (2015) Pelvic Inflammatory Disease. New England Journal of Medicine, 372, 2039-2048. https://doi.org/10.1056/NEJMra1411426

[2] Soper, D.E. (2010) Pelvic Inflammatory Disease. Obstetrics \& Gynecology, 116, 419-428. https://doi.org/10.1097/AOG.0b013e3181e92c54

[3] World Health Organization (1996) Special Program on Research Development and Research Training in Human Reproduction. World Health Organization, Geneva.

[4] Gradison, M. (2012) Pelvic Inflammatory Disease. American Family Physician, 85, 791-796.

[5] Rosenfield, A. and Fathalla, M.F. (2004) The FIGO Manual of Human Reproduction. International Journal of Gynecology \& Obstetrics, 86, 264-266. https://doi.org/10.1016/j.ijgo.2004.04.019

[6] Gul, S., Khalid, B., Albati, Z.A., Ismaeel Badr, R.R., Alfaraj, Z.M., Almatrafi, A.S., et al. (2018) Pelvic Inflammatory Disease. Egyptian Journal of Hospital Medicine, 70.

[7] Washington, A.E., Gove, S., Schachter, J. and Sweet, R.L. (1985) Oral Contraceptives, Chlamydia Trachomatis Infection, and Pelvic Inflammatory Disease: A Word of Caution about Protection. JAMA, 253, 2246-2250. https://doi.org/10.1001/jama.1985.03350390088032 
[8] Mitchell, C. and Prabhu, M. (2013) Pelvic Inflammatory Disease: Current Concepts in Pathogenesis, Diagnosis and Treatment. Infectious Disease Clinics of North America, 27, 793-809. https://doi.org/10.1016/j.idc.2013.08.004

[9] Hubacher, D., Grimes, D.A. and Gemzell-Danielsson, K. (2013) Pitfalls of Research Linking the Intrauterine Device to Pelvic Inflammatory Disease. Obstetrics \& Gynecology, 121, 1091-1098. https://doi.org/10.1097/AOG.0b013e31828ac03a

[10] Shah, H.N., Patel, S. and Nagpal, S. (1978) Pelvic Inflammation (a Study of 800 Cases). Journal of Obstetrics and Gynaecology of India, 28, 429-435.

[11] Trafder, R.L. (1997) A Clinical Study on Chronic PID. MS Thesis, BSMMU, Bangladesh.

[12] St-John, R.K., Brown, S.T. and Tyler Jr., C.W. (1980) Pelvic Inflammatory Disease, 1980. American Journal of Obstetrics and Gynecology, 138, 845. https://doi.org/10.1016/0002-9378(80)91068-6

[13] Sultana, S. (1996) Aetiological Aspect of Chronic PID. A Reported of 349 Case DMCH. Dissertation, BCPS, Bangladesh.

[14] Peterson, H.B., Galaid, E.I. and Cates, W. (1990) Pelvic Inflammatory Disease. Medical Clinics of North America, 74, 1603-1615. https://doi.org/10.1016/S0025-7125(16)30497-7

[15] De Vries, R., Van Bergen, J.E., Postma, M.J. and Pilot-CT Study Group (2006) Systematic Screening for Chlamydia Trachomatis: Estimating cost-effectiveness using Dynamic Modeling and Dutch Data. Value in Health, 9, 1. https://doi.org/10.1111/j.1524-4733.2006.00075.x

[16] Kocher, M. (1980) Review of Pelvic Inflammatory Disease at Kusturba Hospital Delhi. International Symposium of PID Centre for Disease Control, Atlanta, 1-3 April 1980.

[17] Sciarra, J.J. (1994) Infertility: A Global Perspective. The Role of Pelvic Infection. ORGYN: Organon's Magazine on Women \& Health, No. 3, 12-15.

[18] MacLean, A. (1995) Dewhurst's Textbook of Obstetrics and Gynaecology for Postgraduates? 5th Edition, Wiley-Blackwell, Hoboken, 562-567.

[19] Senanayake, P. and Kramer, D.G. (1980) Contraception and the Etiology of Pelvic Inflammatory Disease: New Perspectives. American Journal of Obstetrics and $\mathrm{Gy}^{-}$ necology, 138, 852-860. https://doi.org/10.1016/0002-9378(80)91070-4

[20] Tepper, N.K., Steenland, M.W., Gaffield, M.E., Marchbanks, P.A. and Curtis, K.M. (2013) Retention of Intrauterine Devices in Women Who Acquire Pelvic Inflammatory Disease: A Systematic Review. Contraception, 87, 655-660. https://doi.org/10.1016/j.contraception.2012.08.011 